\title{
MAGIC observations of the microquasar V404 Cygni during the 2015 outburst
}

M. L. Ahnen, ${ }^{1}$ S. Ansoldi, ${ }^{2,3}$ L. A. Antonelli, ${ }^{4}$ C. Arcaro, ${ }^{5}$ A. Babić, ${ }^{6}$ B. Banerjee, ${ }^{7}$ P. Bangale, ${ }^{8}$ U. Barres de Almeida, ${ }^{8}$ J. A. Barrio, ${ }^{9}$ J. Becerra González, ${ }^{10,11}$ W. Bednarek, ${ }^{12}$ E. Bernardini, ${ }^{13,14}$ A. Berti, ${ }^{2,15}$ B. Biasuzzi, ${ }^{2}$ A. Biland, ${ }^{1}$ O. Blanch, ${ }^{3}$ S. Bonnefoy, ${ }^{9}$ G. Bonnoli, ${ }^{16}$ R. Carosi, ${ }^{16}$ A. Carosi,${ }^{4}$ A. Chatterjee, ${ }^{7}$ P. Colin, ${ }^{8}$ E. Colombo, ${ }^{10,11}$ J. L. Contreras, ${ }^{9}$ J. Cortina, ${ }^{3}$ S. Covino,${ }^{4}$ P. Cumani, ${ }^{3}$ P. Da Vela, ${ }^{16}$ F. Dazzi,${ }^{4}$ A. De Angelis, ${ }^{5}$ B. De Lotto,${ }^{2}$ E. de Oña Wilhelmi, ${ }^{17}$ F. Di Pierro, ${ }^{5}$ M. Doert, ${ }^{18}$ A. Domínguez, ${ }^{9}$ D. Dominis Prester, ${ }^{6}$ D. Dorner, ${ }^{19}$ M. Doro,${ }^{5}$ S. Einecke, ${ }^{18}$ D. Eisenacher Glawion, ${ }^{19}$ D. Elsaesser, ${ }^{18}$ M. Engelkemeier, ${ }^{18}$ V. Fallah Ramazani, ${ }^{20}$ A. Fernández-Barral, ${ }^{3 \star}$ D. Fidalgo, ${ }^{9}$ M. V. Fonseca, ${ }^{9}$ L. Font,${ }^{21}$ C. Fruck, ${ }^{8}$ D. Galindo, ${ }^{22}$ R. J. García López, ${ }^{10,11}$ M. Garczarczyk, ${ }^{13}$ M. Gaug, ${ }^{21}$ P. Giammaria, ${ }^{4}$ N. Godinović, ${ }^{6}$ D. Gora, ${ }^{13}$ S. Griffiths, ${ }^{3}$ D. Guberman, ${ }^{3}$ D. Hadasch, ${ }^{23}$ A. Hahn, ${ }^{8}$ T. Hassan, ${ }^{3}$ M. Hayashida, ${ }^{23}$ J. Herrera, ${ }^{10,11}$ J. Hose, ${ }^{8}$ D. Hrupec, ${ }^{6}$ G. Hughes, ${ }^{1}$ K. Ishio,${ }^{8}$ Y. Konno, ${ }^{23}$ H. Kubo, ${ }^{23}$ J. Kushida, ${ }^{23}$ D. Kuveždić, ${ }^{6}$ D. Lelas, ${ }^{6}$ E. Lindfors,${ }^{20}$ S. Lombardi, ${ }^{4}$ F. Longo,${ }^{2,15}$ M. López, ${ }^{9}$ C. Maggio, ${ }^{21}$ P. Majumdar, ${ }^{7}$ M. Makariev, ${ }^{24}$ G. Maneva, ${ }^{24}$ M. Manganaro, ${ }^{10,11}$ K. Mannheim, ${ }^{19}$ L. Maraschi, ${ }^{4}$ M. Mariotti, ${ }^{5}$ M. Martínez, ${ }^{3}$ D. Mazin,,${ }^{8,23}$ U. Menzel, ${ }^{8}$ M. Minev, ${ }^{24}$ R. Mirzoyan, ${ }^{8}$ A. Moralejo, ${ }^{3}$ V. Moreno,${ }^{21}$ E. Moretti, ${ }^{8 \star}$ V. Neustroev, ${ }^{20}$ A. Niedzwiecki, ${ }^{12}$ M. Nievas Rosillo, ${ }^{9}$ K. Nilsson, ${ }^{20,25}$ D. Ninci, ${ }^{3}$ K. Nishijima, ${ }^{23}$ K. Noda, ${ }^{3}$ L. Nogués,${ }^{3}$ S. Paiano, ${ }^{5}$ J. Palacio, ${ }^{3}$ D. Paneque, ${ }^{8}$ R. Paoletti, ${ }^{16}$ J. M. Paredes, ${ }^{22}$ X. Paredes-Fortuny, ${ }^{22}$ G. Pedaletti, ${ }^{13}$ M. Peresano, ${ }^{2}$ L. Perri, ${ }^{4}$ M. Persic, ${ }^{2,4}$ P. G. Prada Moroni, ${ }^{26}$ E. Prandini, ${ }^{5}$ I. Puljak, ${ }^{6}$ J. R. Garcia, ${ }^{8}$ I. Reichardt,${ }^{5}$ W. Rhode, ${ }^{18}$ M. Ribó, ${ }^{22}$ J. Rico,${ }^{3}$ T. Saito, ${ }^{23}$ K. Satalecka, ${ }^{13}$ S. Schroeder, ${ }^{18}$ T. Schweizer, ${ }^{8}$ A. Sillanpää, ${ }^{20}$ J. Sitarek, ${ }^{12}$ I. Šnidarić, ${ }^{6}$ D. Sobczynska, ${ }^{12}$ A. Stamerra, ${ }^{4}$ M. Strzys,${ }^{8}$ T. Surić,${ }^{6}$ L. Takalo, ${ }^{18}$ F. Tavecchio,${ }^{4}$ P. Temnikov, ${ }^{22}$ T. Terzić, ${ }^{6}$ D. Tescaro, ${ }^{5}$ M. Teshima,,${ }^{8,23}$ D. F. Torres,${ }^{27}$ N. Torres-Albà, ${ }^{22}$ A. Treves, ${ }^{2}$ G. Vanzo, ${ }^{10,11}$ M. Vazquez Acosta, ${ }^{10,11}$ I. Vovk, ${ }^{8}$ J. E. Ward, ${ }^{3}$ M. Will, ${ }^{10,11}$ D. Zarić, ${ }^{6}$ (The MAGIC Collaboration), ${ }^{6}$ A. Loh $^{28}$ and J. Rodriguez ${ }^{28}$

Affiliations are listed at the end of the paper 


\begin{abstract}
The microquasar V404 Cygni underwent a series of outbursts in 2015, June 15-31, during which its flux in hard X-rays $(20-40 \mathrm{keV})$ reached about 40 times the Crab nebula flux. Because of the exceptional interest of the flaring activity from this source, observations at several wavelengths were conducted. The MAGIC telescopes, triggered by the INTEGRAL alerts, followed-up the flaring source for several nights during the period June 18-27, for more than $10 \mathrm{~h}$. One hour of observation was simultaneously conducted on a giant $22 \mathrm{GHz}$ radio flare and a hint of signal at GeV energies seen by Fermi-LAT. The MAGIC observations did not show significant emission in any of the analysed time intervals. The derived flux upper limit, in the energy range $200-1250 \mathrm{GeV}$, is $4.8 \times 10^{-12}$ photons $\mathrm{cm}^{-2} \mathrm{~s}^{-1}$. We estimate the gamma-ray opacity during the flaring period, which along with our non-detection points to an inefficient acceleration in the V404 Cyg jets if a very high energy emitter is located further than $1 \times 10^{10} \mathrm{~cm}$ from the compact object.
\end{abstract}

Key words: stars: individual: V404 Cygni (V404 Cyg) - gamma-rays: general - X-rays: binaries.

\section{INTRODUCTION}

The microquasar V404 Cygni (V404 Cyg), located at a parallax distance of $2.39 \pm 0.14 \mathrm{kpc}$ (Miller-Jones et al. 2009), is a binary system of an accreting stellar-mass black hole from a companion star. The black hole mass estimation ranges from about 8 to $15 \mathrm{M}_{\odot}$, while the companion star mass is $0.7_{-0.2}^{+0.3} \mathrm{M}_{\odot}$ (Casares \& Charles 1994; Shahbaz et al. 1994; Khargharia, Froning \& Robinson 2010). The system inclination angle is $67^{\circ}{ }_{-1}^{+3}$ (Shahbaz et al. 1994; Khargharia et al. 2010) and the system orbital period is $6.5 \mathrm{~d}$ (Casares \& Charles 1994). This low-mass X-ray binary (LMXB) showed at least four periods of outbursting activity: the one that led to its discovery in 1989 detected by the Ginga X-ray satellite (Makino et al. 1989), two previous ones in 1938 and 1956 observed in optical and later associated with V404 Cyg (Richter 1989), and the latest in 2015.

In 2015 June, the system underwent an exceptional flaring episode. From the 15th to the end of June the bursting activity was registered by several hard X-ray satellites, such as Swift and INTEGRAL (Barthelmy et al. 2015; Ferrigno et al. 2015). It reached a flux about 40 times larger than the Crab nebula one in the $20-40 \mathrm{keV}$ energy band (Rodriguez et al. 2015). The alerts from these instruments triggered follow-up observations from many other instruments from radio (Mooley et al. 2015; Trushkin et al. 2015b) to very high energies (Archer et al. 2016). Recently, Siegert et al. (2016) claimed the detection of the $511 \mathrm{keV}$ gamma signal from electron-positron annihilation in the June V404 Cyg outburst. In agreement with the models, the variability of the annihilation component suggests that it is produced in the hot plasma situated in the inner parts of the accretion disc (the so-called corona). On the other hand, the possible excess seen in the Fermi-LAT (Loh et al. 2016), in temporal coincidence with a giant radio flare (Trushkin et al. 2015b), suggests that the $\mathrm{HE}$ emission, in the $\mathrm{MeV}-\mathrm{GeV}$ energy range, originates inside the relativistic jet. Furthermore, the observations of an orphan flare in the near-infrared (Tanaka et al. 2016) and the fast variability of the optical polarization (Lipunov et al. 2016; Shahbaz et al. 2016) indicate the presence of a jet. Tanaka et al. (2016) derive the jet parameters, like the magnetic field, and constrain the emission zone.

Very high energy (VHE; $E \gtrsim 50 \mathrm{GeV}$ ) gamma-ray emission from microquasars has been theoretically predicted in association with the jets where relativistic particles are accelerated. VHE radiation could be produced via leptonic (e.g. Bosch-Ramon, Romero \&
Paredes 2006) or hadronic processes (e.g. Romero et al. 2003). The IC process on photons from the companion star was proposed as the most likely scenario in the case of two microquasars detected in the HE regime: the high-mass X-ray binaries Cygnus X-1 (Zanin et al. 2016; Zdziarski et al. 2016) and Cygnus X-3 (Abdo et al. 2009; Tavani et al. 2009). In the case of the possible HE detection of the high-mass X-ray binary SS433 (Bordas et al. 2015), the proposed emission mechanism is hadronic via proton-proton collisions. On the other hand, LMXBs, composed of cold and old stars, do not provide a proper photon field target for this process to take place. In LMXBs, the dominant processes in the leptonic scenario are synchrotron and synchrotron self-Compton emissions from an extended dissipation region in the jet (Zhang et al. 2015). Differently from HMXBs where the dense matter environments favour emission from neutral pion decay (Bosch-Ramon \& Khangulyan 2009), in LMXBs the donor star presents weak winds. Therefore in the hadronic scenario, photopion production could be considered as the emission mechanism instead (Levinson \& Waxman 2001). In the innermost dissipation region of the jet, photopions are produced at the $\Delta$ resonance by the interaction of accelerated protons and external $\mathrm{X}$-ray photons entering the jet. Given the lack of targets provided by the low-mass companion star in LMXB (like V404 Cyg), gamma rays are expected to be produced inside the relativistic jets and in particular where they are most compact, like at their base. According to models, gamma rays are created by the interaction of the particles in the jet with the radiation and magnetic fields in the jet itself (see e.g. Bosch-Ramon et al. 2006; Vila \& Romero 2008; Vieyro \& Romero 2012).

Triggered by the INTEGRAL alerts, MAGIC observed V404 Cyg for several nights between 2015 June 18 and 27, collecting data for more than $10 \mathrm{~h}$. In Section 2, we present the observations and the instrument overview. The analysis of the night-wise observations and the focused analysis following the INTEGRAL light curve are presented in Section 3. Finally, we discuss the possible physical implication of the results of the MAGIC observations in Section 4.

\section{OBSERVATIONS AND DATA ANALYSIS}

MAGIC is a stereoscopic system of two $17 \mathrm{~m}$ diameter Imaging Atmospheric Cherenkov Telescopes (IACT). It is located at $2200 \mathrm{~m}$ a.s.l. in the El Roque de los Muchachos Observatory, La Palma, Spain. The performance of the telescopes is described in Aleksić et al. (2016): the trigger threshold is $\sim 50 \mathrm{GeV}$ below $30^{\circ}$ zenith and 


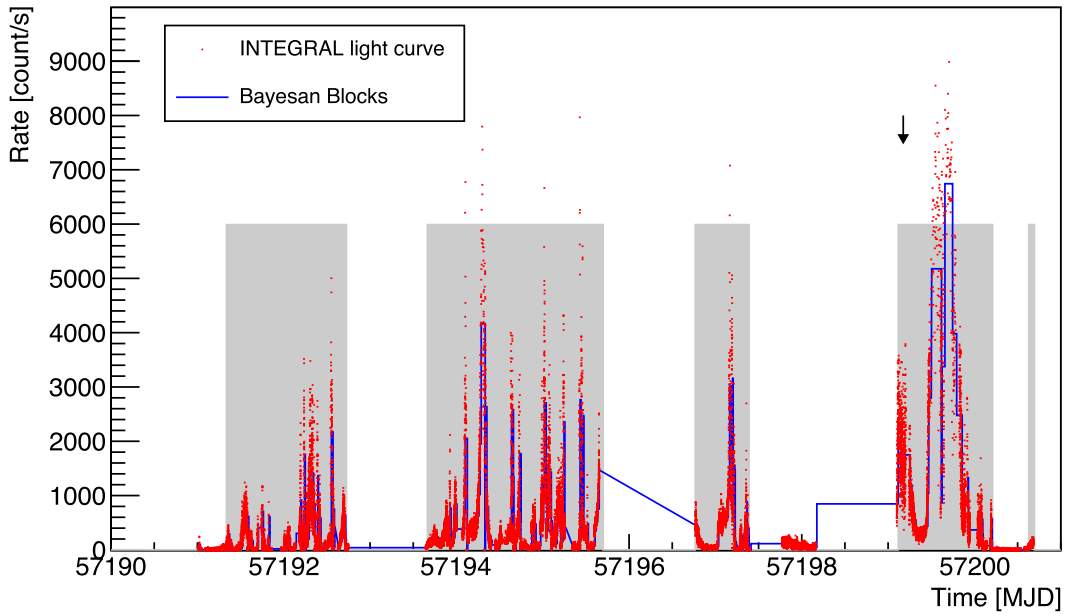

Figure 1. INTEGRAL light curve (red points) in the energy range $20-40 \mathrm{keV}$ with the definition of the flaring interval. The time intervals with the highest flaring activity (grey bands) used in the analysis of MAGIC data are defined following the Bayesian block method. The arrow refers to the peak of the Fermi-LAT hint of signal.

the integral sensitivity is $0.66 \pm 0.03$ per cent of the Crab nebula flux above $220 \mathrm{GeV}$ in $50 \mathrm{~h}$ of observations.

Most of the MAGIC observations were triggered by the INTEGRAL alerts sent via the Gamma-ray Coordinate Network (GCN). The first alert was received at 00:08:39 UT on June 18. MAGIC observations continued until June 27 when the INTEGRAL alerts ceased. On the night between June 22 and 23, the observations were not triggered by any alert, but scheduled a priori according to a multiwavelength campaign on the V404 Cyg system. The rest of the observations followed a GCN alert processed by the MAGIC Gamma-Ray Burst procedure. This procedure allows an automatic and fast re-pointing of the telescopes to the burst position in $\sim 20 \mathrm{~s}$. Most of the observations were performed during the strongest hard X-ray flares. In total, MAGIC observed the microquasar for eight non-consecutive nights collecting more than $10 \mathrm{~h}$ of data, some coinciding with observations at other energies.

The data were analysed using the MAGIC software, MARS (Zanin et al. 2013), version 2-16-0. Standard event cuts are used to improve the signal-to-background ratio in the MAGIC data as described in Aleksić et al. (2016). The selections applied to estimate the significance of the source are based on hadronness, $\Theta^{2}$ and on the size of the shower images. The hadronness is a variable to quantify how likely is that a given event was produced by a hadronic atmospheric shower, while the $\Theta$ is the angular distance of each event from the position of the source in the camera plane.

\section{RESULTS}

To avoid an iterative search over different time bins, we assumed that the TeV flares were simultaneous to the X-ray ones. We defined the time intervals where we search for signals in the MAGIC data, to match those of the flares in the INTEGRAL light curve. We analysed the INTEGRAL-IBIS data (20-40 keV) publicly available with the osa software version $10.2,{ }^{1}$ obtaining the light curve shown in Fig. 1.

The time selection for the MAGIC analysis was performed running a Bayesian block (Scargle et al. 2013) analysis on the INTEGRAL light curve (see Fig. 1). The Bayesian block analysis is meant

\footnotetext{
${ }^{1} \mathrm{http}: / / \mathrm{www}$. isdc.unige.ch/integral/analysis
}

Table 1. Time intervals selected by the Bayesian block algorithm. The start and stop times are in MJD.

\begin{tabular}{lc}
\hline Start & Stop \\
\hline 57191.337 & 57192.725 \\
57193.665 & 57195.700 \\
57196.765 & 57197.389 \\
57199.116 & 57200.212 \\
57200.628 & 57200.695 \\
\hline
\end{tabular}

to identify structures in a time series and to divide these features into adaptive time bins called blocks. To partition the light curve, the algorithm (Jackson et al. 2015) maximizes a quantity that describes how well a constant flux represents the data in a given block. Once the blocks are defined we grouped them into intervals that describe each flaring period. The analysis did not single out periods with distinctively high levels of source activity. The Bayesian blocks used to determine the limits of the periods of activity are listed in Table 1. This analysis selected in total about $7 \mathrm{~h}$ out of the $10 \mathrm{~h}$ observed.

We searched for VHE gamma-ray emission stacking the MAGIC data of the selected time intervals $(\sim 7 \mathrm{~h})$. We found no significant emission in the $\sim 7 \mathrm{~h}$ sample (see Fig. 2). We found no significant emission also in any of the sub-samples considered (See Table 2). We then computed integral (see Table 2) and differential upper limits (ULs) (see Fig. 3) for the observations assuming a powerlaw spectral shape of index -2.6 . The Li \& Ma method ( $\mathrm{Li} \&$ Ma 1983) was used to estimate the detection significance while the Rolke method (Rolke, López \& Conrad 2005) was used for the computation of the ULs. The ULs were computed using a Poisson distribution for the background, requiring a 95 per cent confidence level and considering a 30 per cent systematic uncertainty.

Loh et al. (2016) found in the Fermi-LAT data evidences for a detection above $4 \sigma$ of a source centred $0.65 \mathrm{deg}$ - which is within 95 per cent of the PSF - away from V404 Cyg and temporally coincident with the brightest radio and hard X-ray flare of this source. The Fermi-LAT signal is found in the $0.1-100 \mathrm{GeV}$ energy interval and it peaks at MJD 57199.21 \pm 0.12 . MAGIC observation during this period starts at MJD 57199.15 and lasts up to MJD 57199.20, which is within the interval of the Fermi-LAT 


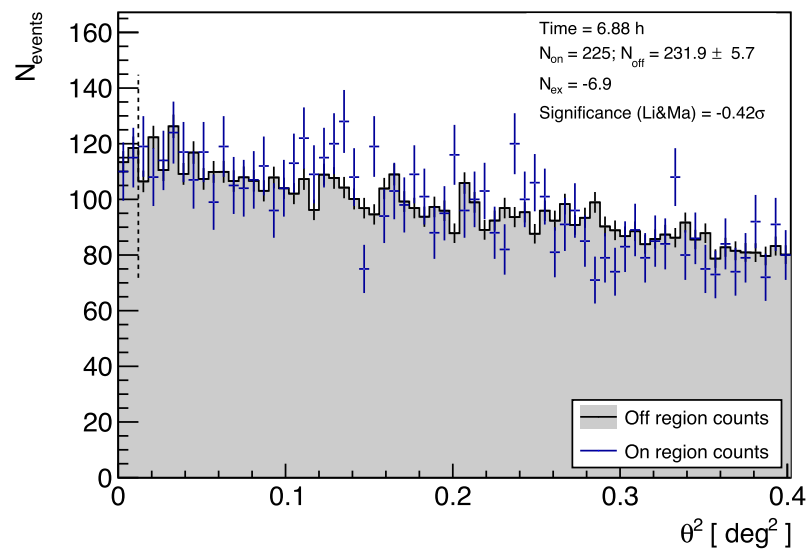

Figure 2. Distribution of the square of the angular distance from the position of the source. In the $7 \mathrm{~h}$ accumulation there is no evidence of signal from V404 Cyg in the MAGIC data.

excess. For this data set we re-computed the differential ULs using a power law with index -3.5 (see green UL in Fig. 3) according to the LAT analysis presented in Loh et al. (2016). The MAGIC ULs are two orders of magnitude higher than the extrapolation of the Fermi-LAT spectrum (see Fig. 3).

\section{DISCUSSION}

MAGIC observed V404 Cyg for several nights during an outbursting period for a total amount of about $10 \mathrm{~h}$. The analysis of the data resulted in a non-detection and both differential and integral ULs have been computed. The luminosity ULs calculated for the full observation period, considering the source at a distance of $2.4 \mathrm{kpc}$, is $\sim 2 \times 10^{33} \mathrm{erg} \mathrm{s}^{-1}$, in contrast with the extreme luminosity emitted in the X-ray band $\left(\sim 2 \times 10^{38} \mathrm{erg} \mathrm{s}^{-1}\right.$; Rodriguez et al. 2015) and other wavelengths.

The emission of microquasars at VHE is still under debate. Processes similar to those taking place in AGNs also occur in microquasars, but at a quite different scale. Similar to quasars, microquasars develop jets, possibly relativistic, at least in their X-ray hard state (Fender, Belloni \& Gallo 2004). If the acceleration that takes place in the jets is efficient enough, VHE photon fluxes could reach $10^{-13}$ to $10^{-12}$ photons $\mathrm{cm}^{-2} \mathrm{~s}^{-1}$ (for an object at about $5 \mathrm{kpc}$ ) (Bosch-Ramon et al. 2006; Khiali et al. 2015; Zhang et al. 2015) making them detectable by this or next generation of IACT.

During the 2015 June outburst of V404 Cyg, there are convincing evidence of jet emission given by the optical observations (Lipunov et al. 2016; Shahbaz et al. 2016; Tanaka et al. 2016). In particular on June 26, a hint of detection $(\sim 4 \sigma)$ in the Fermi-LAT data has been reported by Loh et al. (2016). Moreover, the presence of a giant radio flare (Trushkin et al. 2015b), an increase of the hardness ratio in the X-ray band (Loh et al. 2016) and optical fast variability (Gandhi et al. 2016) indicate that the jet environment dramatically changed on that day.

MAGIC conducted an extensive campaign dedicated to this source, which includes $1 \mathrm{~h}$ of simultaneous observations with the Fermi-LAT excess. No signal was detected in any of the time intervals considered. We set an energy flux UL from a selected data set of about $\sim 7 \mathrm{~h}$ of $\sim 2.9 \times 10^{-12} \mathrm{erg} \mathrm{cm}^{-2} \mathrm{~s}^{-1}$. The UL is about two orders of magnitude smaller than the flux released in the $\mathrm{GeV}$ regime $\sim 4.2 \times 10^{-10} \mathrm{erg} \mathrm{cm}^{-2} \mathrm{~s}^{-1}$ (Loh et al. 2016). Tanaka et al. (2016) modelled the spectrum of the jet emission in the case of V404 Cyg, obtaining a total radiated flux of $F_{\text {rad }}=1.015 \times 10^{-7} \mathrm{erg} \mathrm{cm}^{-2} \mathrm{~s}^{-1}$. We compare the flux UL obtained from our data with the total radiated flux from this model: the resulting efficiency for VHE gamma emission is lower than 0.003 per cent.

Models predict $\mathrm{TeV}$ emission from this type of systems under efficient particle acceleration on the jets (Atoyan \& Aharonian 1999; Zhang et al. 2015) or strong hadronic jet component (Vila \& Romero 2008). If produced, VHE gamma rays may annihilate via pair creation in the vicinity of the emitting region. For gamma rays in an energy range between $200 \mathrm{GeV}$ and $1.25 \mathrm{TeV}$, the largest cross-section occurs with NIR photons. For a low-mass microquasar, like V404 Cyg, the contribution of the NIR photon field from the companion star (with a bolometric luminosity of $\sim 10^{32} \mathrm{erg} \mathrm{s}^{-1}$ ) is very low. During the period of flaring activity, disc and jet contributions are expected to dominate. During the outburst activity of 2015 June, the magnitude of the $K$-band reached $m=10.4$ (Shaw et al. 2015), leading to a luminosity on the NIR regime of $L_{\mathrm{NIR}}=v \phi_{m=0} 4 \pi d^{2} 10^{-m / 2.5}=4.1 \times 10^{34} \mathrm{erg} \mathrm{s}^{-1}$, where $v$ is the frequency for the $2.2 \mu \mathrm{m} \mathrm{K}$ band, $\phi_{m=0}=670 \mathrm{Jy}$ is the $K$-band reference flux and $d=2.4 \mathrm{kpc}$ is the distance to the source. The detected NIR radiation from V404 Cyg during this flaring period was expected to be dominated by optically thick synchrotron emission from the jet or to be originated inside the accretion flow, given the lack of evidence of polarization (Tanaka et al. 2016). Consequently, stronger gamma-ray absorption is expected at the base of the jets. The gamma-ray opacity due to NIR radiation inside V404 Cyg can be estimated as $\tau_{\gamma \gamma} \sim \sigma_{\gamma \gamma} n_{\mathrm{NIR}} r$, given by Aharonian et al. (2005). The cross-section of the interaction is defined by $\sigma_{\gamma \gamma}$, whose value is $\sim 1 \times 10^{-25} \mathrm{~cm}^{2}$. The NIR photon density is calculated as $n_{\mathrm{NIR}}=L_{\mathrm{NIR}} / \pi r^{2} c \epsilon$, where $r$ is the radius of the jet where NIR photons are expected to be emitted, $c$ is the speed of light

Table 2. MAGIC observation periods of V404 Cyg. For each night the observation interval and duration are reported together with the detection significance for that night. In the last column the integral flux ULs for energies between 200 and $1250 \mathrm{GeV}$ are reported. The last row reports the same quantities for the periods selected with the Bayesian block algorithm.

\begin{tabular}{lcccc}
\hline $\begin{array}{l}\text { Observation date } \\
\text { (June 2015) }\end{array}$ & Observation MJD & $\begin{array}{c}\text { Effective time } \\
(\mathrm{h})\end{array}$ & $\begin{array}{c}\text { Detection } \\
\text { significance } \\
(\sigma)\end{array}$ & $\begin{array}{c}\text { Flux UL } \\
(200<E<1250 \mathrm{GeV}) \\
\left(\mathrm{photons} \mathrm{cm}^{-2} \mathrm{~s}^{-1}\right)\end{array}$ \\
\hline 18th & $57191.006-57191.146$ & 2.99 & -0.43 & $5.1 \times 10^{-12}$ \\
19 th & $57191.960-57192.055$ & 1.9 & -0.6 & $1.00 \times 10^{-11}$ \\
$21 \mathrm{st}$ & $57193.997-57194.025$ & 0.66 & 1.57 & $4.35 \times 10^{-11}$ \\
22nd & $57195.021-57195.04957195 .103-57195.134$ & 1.33 & 0.09 & $1.67 \times 10^{-11}$ \\
23rd & $57196.003-57196.124$ & 2.74 & -0.45 & $3.7 \times 10^{-12}$ \\
26th & $57199.158-57199.204$ & 1.03 & -1.41 & $6.6 \times 10^{-12}$ \\
27th & $57200.085-57200.11557200 .144-57200.202$ & 1.97 & -0.57 & $1.23 \times 10^{-11}$ \\
Selected & See Table 1 & 6.88 & -0.42 & $4.8 \times 10^{-12}$ \\
\hline
\end{tabular}




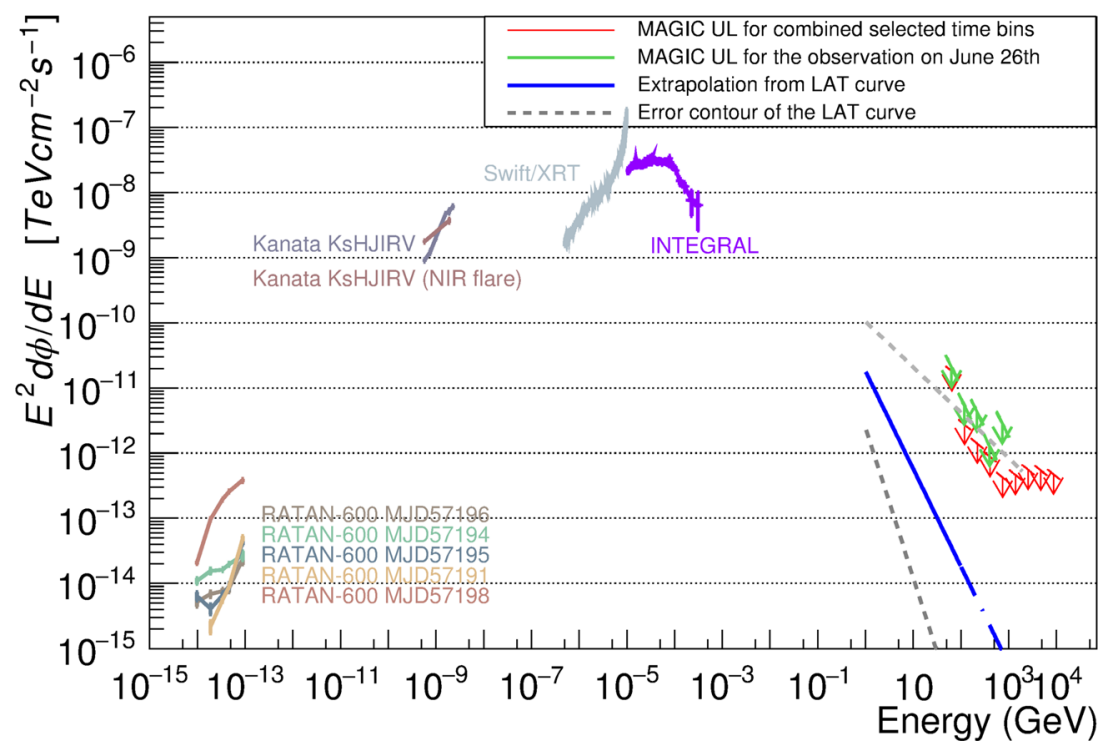

Figure 3. Multiwavelength spectral energy distribution of V404 Cyg during the 2015 June flaring period. In red, MAGIC ULs are given for the combined Bayesian block time bins $(\sim 7 \mathrm{~h})$ for which a power-law function with a photon index of 2.6 was assumed. In green, MAGIC ULs for observations on June 26 th, simultaneously taken with the Fermi-LAT hint (Loh et al. 2016). In this case, a photon index of 3.5 was applied following Fermi-LAT results. All the MAGIC ULs are calculated for a 95 per cent confidence level, also considering a 30 per cent systematic uncertainty. The extrapolation of the Fermi-LAT spectrum is shown in blue with $1 \sigma$ contour (grey dashed lines). In the X-ray regime, INTEGRAL (20-40 keV; Rodriguez et al. 2015) and Swift-XRT (0.2-10 keV; Tanaka et al. 2016) data are depicted. At lower energies, Kanata-HONIR optical and NIR data are shown, taken from Tanaka et al. (2016). Finally, RATAN-600 radio data, from Trushkin et al. (2015a), are presented for different days during the flaring period.

and $\epsilon \sim 1 \times 10^{-12} \mathrm{erg}$ is the energy of the target photon field. Assuming the aforementioned luminosity of $L_{\mathrm{NIR}}=4.1 \times 10^{34} \mathrm{erg} \mathrm{s}^{-1}$, the gamma-ray opacity at a typical radius $r \sim 1 \times 10^{10} \mathrm{~cm}$ may be relevant enough to avoid VHE emission above $200 \mathrm{GeV}$. Moreover, if IC on X-rays at the base of the jets $\left(r \lesssim 1 \times 10^{10} \mathrm{~cm}\right)$ is produced, this could already prevent electrons to reach the $\mathrm{TeV}$ regime, unless the particle acceleration rate in V404 Cyg is close to the maximum achievable including specific magnetic field conditions (see e.g. Khangulyan, Aharonian \& Bosch-Ramon 2008). On the other hand, VHE photon absorption becomes negligible for $r>1 \times 10^{10} \mathrm{~cm}$. Thus, if the VHE emission is produced in the same region as HE radiation $\left(r \gtrsim 1 \times 10^{11} \mathrm{~cm}\right.$, to avoid $\mathrm{HE}$ photon absorption in the X-ray photon field), then it would not be significantly affected by pair production attenuation $\left(\sigma_{\gamma \gamma}<1\right)$. Therefore a VHE emitter at $r \gtrsim 1 \times 10^{10} \mathrm{~cm}$, along the non-detection by MAGIC, suggests either a low particle acceleration rate inside the V404 Cyg jets or not enough energetics of the VHE emitter.

\section{ACKNOWLEDGEMENTS}

We are grateful to Lucia Pavan at UNIGE for the support on the INTEGRAL-IBIS data analysis. We would like to thank Dr. Rodriguez and Prof. Tanaka for providing the multiwavelength data. We thank the anonymous reviewer for the useful comments that helped to set our results in a broader multiwavelength context. We would like to thank the Instituto de Astrofísica de Canarias for the excellent working conditions at the Observatorio del Roque de los Muchachos in La Palma. The financial support of the German BMBF and MPG, the Italian INFN and INAF, the Swiss National Fund SNF, the ERDF under the Spanish MINECO (FPA2015-69818-P, FPA2012-36668, FPA2015-68378-P, FPA2015-69210-C6-2-R, FPA2015-69210-C64-R, FPA2015-69210-C6-6-R, AYA2015-71042-P, AYA201676012-C3-1-P, ESP2015-71662-C2-2-P, CSD2009-00064) and the
Japanese JSPS and MEXT is gratefully acknowledged. This work was also supported by the Spanish Centro de Excelencia 'Severo Ochoa' SEV-2012-0234 and SEV-2015-0548, and Unidad de Excelencia 'María de Maeztu' MDM-2014-0369, by the Croatian Science Foundation (HrZZ) Project 09/176 and the University of Rijeka Project 13.12.1.3.02, by the DFG Collaborative Research Centers SFB823/C4 and SFB876/C3, and by the Polish MNiSzW grant 2016/22/M/ST9/00382.

\section{REFERENCES}

Abdo A. A. et al., 2009, Science, 326, 1512

Aharonian F. et al., 2005, Science, 309, 746

Albert et al., 2007, ApJ, 665, L51

Albert et al., 2008, Nucl. Instrum. Methods Phys. Res. A, 588, 424

Aleksić et al., 2016, Astropart. Phys., 72, 76

Archer A. et al., 2016, ApJ, 831, 113

Atoyan A. M., Aharonian F. A., 1999, MNRAS, 302, 253

Barthelmy S. D., D'Ai A., D'Avanzo P., Krimm H. A., Lien A. Y., Marshall

F. E., Maselli A., Siegel M. H., 2015, GCN Circ., 17929, 1

Bordas P., Yang R., Kafexhiu E., Aharonian F., 2015, ApJ, 807, L8

Bosch-Ramon V., Khangulyan D., 2009, Int. J. Mod. Phys. D, 18, 347

Bosch-Ramon V., Romero G. E., Paredes J. M., 2006, A\&A, 447, 263

Casares J., Charles P. A., 1994, Evol. X-ray Bin., 308, 107

Fender R. P., Belloni T. M., Gallo E., 2004, MNRAS, 355, 1105

Ferrigno C. et al., 2015, Astron. Telegram, 7662

Gandhi P. et al., 2016, MNRAS, 459, 554

Jackson B. et al., 2005, IEEE Signal Process. Lett., 12, 105

Khangulyan D., Aharonian F., Bosch-Ramon V., 2008, MNRAS, 383, 467

Khargharia J., Froning C. S., Robinson E. L., 2010, ApJ, 716, 1105

Khiali B., de Gouveia Dal Pino E. M., del Valle M. V., 2015, MNRAS, 449, 34

Kimura M. et al., 2016, Nature, 529, 54

Levinson A., Waxman E., 2001, Phys. Rev. Lett., 87, 171101

Li T.-P., Ma Y.-Q., 1983, ApJ, 272, 317

Lipunov V. M. et al., 2016, ApJ, 833, 198 
Loh A. et al., 2016, MNRAS, 462, L111

Makino F., Wagner R. M., Starrfield S., Buie M. W., Bond H. E., Johnson J., Harrison T., Gehrz R. D., 1989, IAU Circ., 4786, 1

Miller-Jones J. C. A., Jonker P. G., Dhawan V., Brisken W., Rupen M. P., Nelemans G. Gallo E., 2009, ApJ, 706, L230

Mooley K., Fender R., Anderson G., Staley T., Kuulkers E., Rumsey C., 2015, Astron. Telegram, 7658

Muñoz-Darias T. et al., 2016, Nature, 534, 75

Richter G. A., 1989, Inf. Bull. Var. Stars, 3362, 1

Rodriguez J. et al., 2015, A\&A, 581, L9

Rolke W. A., López A. M., Conrad J., 2005, Nucl. Instrum. Methods Phys. Res. A, 551, 493

Romero G. E., Torres D. F., Kaufman Bernadó M. M., Mirabel I. F., 2003, A\&A, 410, L1

Scargle J. D. Norris J. P., Jackson B., Chiang J., 2013, ApJ, 764, 167

Shahbaz T. Ringwald F. A., Bunn J. C., Naylor T., Charles P. A., Casares J., 1994, MNRAS, 271, L10

Shahbaz T., Russell D. M., Covino S., Mooley K., Fender R. P., Rumsey C., 2016, MNRAS, 463, 1822

Shaw A. W., Knigge C., Meisenheimer K., Ibanez J. M., 2015, Astron. Telegram, 7738

Siegert T. et al., 2016, Nature, 531, 341

Tanaka Y. T. et al., 2016, ApJ, 823, 35

Tavani M. et al., 2009, Nature, 462, 620

Trushkin S. A., Nizhelskij N. A., Tybulev P. G., 2015a, Astron. Telegram, 7667, 1

Trushkin S. A., Nizhelskij N. A., Tybulev P. G., 2015b, Astron. Telegram, 7716,1

Vieyro F. L., Romero G. E., 2012, A\&A, 542, A7

Vila G. S., Romero G. E., 2008, Int. J. Mod. Phys. D, 17, 1903

Zanin R. et al., 2013, Proc 33rd ICRC, Rio de Janeiro, Brazil, p. 773, 201

Zanin R., Fernández-Barral A., de Oña Wilhelmi E., Aharonian F., Blanch O., Bosch-Ramon V., Galindo D., 2016, A\&A, 596, A55

Zdziarski A. A., Malyshev D., Chernyakova M., Pooley G. G., 2016, MNRAS, in press

Zhang J.-F., Gu W.-M., Liu T., Xue L., Lu J.-F., 2015, ApJ, 806, 168

${ }^{1}$ ETH Zurich, CH-8093 Zurich, Switzerland

${ }^{2}$ Università di Udine, and INFN Trieste, I-33100 Udine, Italy

${ }^{3}$ Institut de Fisica d'Altes Energies (IFAE), The Barcelona Institute of Science and Technology, Campus UAB, E-08193 Bellaterra (Barcelona), Spain

${ }^{4}$ INAF National Institute for Astrophysics, I-00136 Rome, Italy

${ }^{5}$ Università di Padova and INFN, I-35131 Padova, Italy
${ }^{6}$ Croatian MAGIC Consortium, Rudjer Boskovic Institute, University of Rijeka, University of Split - FESB, University of Zagreb-FER, University of Osijek, 21000 Split, Croatia

${ }^{7}$ Saha Institute of Nuclear Physics, 1/AF Bidhannagar, Salt Lake, Sector-1, Kolkata 700064, India

${ }^{8}$ Max-Planck-Institut für Physik, D-80805 München, Germany

${ }^{9}$ Universidad Complutense, Dpto. Física Atómica - Facultad de Ciencias Físicas, E-28040 Madrid, Spain

${ }^{10}$ Inst. de Astrofísica de Canarias, E-38200 La Laguna, Tenerife, Spain

${ }^{11}$ Universidad de La Laguna, Dpto. Astrofísica, E-38206 La Laguna, Tenerife, Spain

${ }^{12}$ University of Łódź, Division of Astrophysics, PL-90236 Lodz, Poland

${ }^{13}$ Deutsches Elektronen-Synchrotron (DESY), D-15738 Zeuthen, Germany

${ }^{14}$ Humboldt University of Berlin, Institut für Physik Newtonstr. 15, D-12489 Berlin, Germany

${ }^{15}$ Also at University of Trieste

${ }^{16}$ Università di Siena, and INFN Pisa, Dipartimento di Fisica, I-53100 Siena, Italy

${ }^{17}$ Institute for Space Sciences (CSIC/IEEC), E-08193 Barcelona, Spain

${ }^{18}$ Technische Universität Dortmund, Fakultät Physik, D-44221 Dortmund, Germany

${ }^{19}$ Universität Würzburg, Fakultät für Physik und Astronomie, D-97074 Würzburg, Germany

${ }^{20}$ Finnish MAGIC Consortium, Tuorla Observatory, University of Turku and Astronomy Division, University of Oulu, FI-21500 Piikkiö, Finland

${ }^{21}$ Unitat de Física de les Radiacions, Departament de Física, and CERESIEEC, Universitat Autònoma de Barcelona, E-08193 Bellaterra, Spain

${ }^{22}$ Universitat de Barcelona, ICC, IEEC-UB, E-08028 Barcelona, Spain

${ }^{23}$ Japanese MAGIC Consortium, ICRR, The University of Tokyo, Department of Physics and Hakubi Center, Kyoto University, Tokai University, The University of Tokushima, Japan 606-8502 Kyoto, Japan

${ }^{24}$ Institute for Nuclear Research and Nuclear Energy, BG-1784 Sofia, Bulgaria

${ }^{25}$ Now at Finnish Centre for Astronomy with ESO (FINCA), Turku, Finland

${ }^{26}$ Università di Pisa, and INFN Pisa, I-56126 Pisa, Italy

${ }^{27}$ ICREA and Institute for Space Sciences (CSIC/IEEC), E-08193 Barcelona, Spain

${ }^{28}$ Laboratoire AIM (CEA/IRFU-CNRS/INSU-University Paris Diderot, FR-91191, Gif-sur-Yvette Cedex

This paper has been typeset from a $\mathrm{T}_{\mathrm{E}} \mathrm{X} / \mathrm{L} \mathrm{T} \mathrm{E} \mathrm{X}$ file prepared by the author. 